\title{
Servir solidariamente, un rasgo esencial del aprendizaje para el desarrollo sostenible desde el compromiso social
}

\section{Serving in Solidarity: An Essential Feature of Learning for Sustainable Development Based on Social Commitment}

\author{
María Teresa Sánchez Reyes \\ Universidad Católica Andrés Bello (ucab) Guayana, Venezuela \\ mtesanchez@gmail.com \\ Damelis del Valle Cermeño Guaina \\ Universidad Católica Andrés Bello (ucab) Guayana, Venezuela \\ cermenodamelis@gmail.com
}

https://doi.org/ 10.48102/didac.2022..79_ENE-JUN.97

8

\section{RESUMEN}

El propósito de este artículo es presentar parcialmente los resultados de una investigación, de la línea educación para el desarrollo sustentable (EDS), desarrollada en la Universidad Católica Andrés Bello (UCAB), aplicados a experiencias vividas. Para esto, se utilizó la teoría pedagógica de la experiencia vivida de Max van Manen, quien ha contribuido de manera notoria en la exploración y la comprensión de los significados esenciales pedagógicos. Para penetrar ese mundo de la vida, se utilizó la fenomenología hermenéutica ( $\mathrm{FH}$ ) como marco metodológico. En este artículo se hace énfasis en las Cátedras de Compromiso Social, las cuales crean conciencia de las vulnerabilidades de la sociedad, la especie y la Casa Común; así surge el Servicio Solidario como un rasgo esencial. Este trabajo es descriptivo y documental.

Palabras clave: Pedagogía ignaciana; educación para el desarrollo sostenible (EDS); ética de la sostenibilidad (ES); experiencia vivida; fenomenología hermenéutica (FH); Servicio Solidario.

\begin{abstract}
The purpose of this article is to partially present the result of an investigation, the Education for Sustainable Development (ESD) line, developed at Andres Bello Catholic University (UCAB), applied to lived experiences. To do this, the pedagogical theory of Max van Manen from the lived experience, who has contributed notably to the exploration and understanding of the essential pedagogical meanings. To penetrate this world of life, the Hermeneutical Phenomenology (HF) is followed as a methodological framework. In this article emphasis is placed on the chairs of Social Commitment, they create awareness of the vulnerabilities of society, the species, and the Common
\end{abstract}


House; thus, the Solidarity Service arises as an essential feature. This work is descriptive and documentary.

Keywords: Ignatian Pedagogy; Education Sustainable Development (ESD); Ethics of Sustainability (ES); Lived Experience (EV); Hermeneutical Phenomenology (FH); Solidarity Service.

Fecha de recepción: 24/05/2021

Fecha de aceptación: 03/09/2021

\section{Introducción}

El propósito de este artículo es compartir los resultados de una investigación desarrollada en la Universidad Católica Andrés Bello (UCAB), titulada "El aprendizaje para el desarrollo sustentable como experiencia vivida en la UCAB. Un acercamiento a un nuevo modelo ético y social”. En esta investigación se identificó el Servicio Solidario como un rasgo esencial en el aprendizaje de la ética de la sostenibilidad (Es) y la intención es traducirlo en un texto fenomenológico (TF) desde las Cátedras de Compromiso Social. La UCAB, de inspiración cristiana, que trabaja la formación integral de sus educandos, diseña estrategias de aprendizaje que conecten a sus educandos con la vida, con su realidad y con su país, en un hacer inter y transdisciplinario, en beneficio de los más necesitados. Esto demanda docentes dispuestos a compartir con diversos sectores sociales, a través de un diálogo interinstitucionalizado, en la búsqueda de una opción ética compartida (Fernández, 2005).

Las Cátedras de Compromiso Social, según el Reglamento 2.59, "se comprometen con comunidades a cuyas obras sociales prestan un servicio continuado a través de las prácticas de los estudiantes" (UCAB, 2020, p. 3); con miras al empoderamiento de competencias actitudinales para el desarrollo sostenible (DS).

Esto es coherente con la declaratoria de valores relacionados con las experiencias educativas que nos ocupan: compromiso social, compromiso por el desarrollo sustentable y conciencia ciudadana, es decir, "formar ciudadanos en la UCAB conduce al compromiso institucional de entender las actitudes, los conocimientos y el ejercicio profesional como medios humanizadores" (UCAB, 2013, p. 33).

A su vez, con la fenomenología hermenéutica $(\mathrm{FH})$ se ingresa en el mundo de la vida del acto edu- cativo, concebido como un proceso humano de carácter ético y relacional. $\mathrm{La} \mathrm{FH}$ es un enfoque metodológico humanista del paradigma naturalista, orientado a la descripción e interpretación de las estructuras esenciales de la experiencia vivida, en pro de arraigar la formación de la persona "total", como lo demandan los objetivos del desarrollo sostenible (oDs).

De manera resumida, en este artículo se exponen el objeto de estudio, su importancia y las preguntas de investigación. Asimismo, se comparte una pincelada de los constructos teóricos. También se incluye un apartado sobre la epistemología de la FH y el procedimiento utilizado en la investigación. Posteriormente, se detallan los resultados del Servicio Solidario en un TF y se muestra un modelo de aprendizaje de una es. Por último, se concluye con algunos aportes a esta línea de investigación.

\section{El fenómeno de estudio y su fundamentación teórica}

El aprendizaje para un humanismo solidario asume la pedagogía como una ciencia humana capaz de estudiar, según Van Manen, "las influencias que están en el núcleo de las interacciones, situaciones y relaciones especiales entre los educadores y los estudiantes, los padres y los hijos" (Ayala, 2011, p. 121). Esto exige una actuación ética pedagógica del docente, más si se pretende formar profesionales integrales.

Kolvenbach (2000) lo resume del siguiente modo: "la persona completa del mañana debe tener una solidaridad bien informada [...], pues la solidaridad se aprende a través del contacto más que de nociones" (p. 8). Así, el futuro profesional de los estudiantes está influenciado por las experiencias vividas que han tocado su corazón y que lo han movilizado a cambiar algunos patrones de comportamiento.

30 - Servir solidariamente, un rasgo esencial del aprendizaje para el desarrollo sostenible desde el compromiso social María Teresa Sánchez Reyes, Damelis del Valle Cermeño Guaina DIDAC 79 (2022): 29-38 
Por otro lado, llama la atención que en estudios realizados en la UCAB la conclusión principal sea: "no es posible apreciar cambios significativos en la actitud que fundamenta la cultura de sustentabilidad ambiental de los estudiantes" (UCAB, 2017; UCAB, 2019), lo cual es un reflejo de las interacciones de la sociedad con su entorno.

Producir conocimientos de acuerdo con la pedagogía ignaciana representa un valioso aporte para las transformaciones humanas deseadas. Kolvenbach (2000) señala que los estudiantes en su formación deben impregnarse de la realidad de este mundo, que "aprendan a sentirlo, a pensarlo críticamente, a responder a sus sufrimientos y a comprometerse con él de forma constructiva" (p. 8).

Es decir, de forma general, se aspira a trascender los significados ético-relacionales de las experiencias vividas en el proceso de aprendizaje de la Es.

\section{Propósito e interrogantes}

El propósito de este artículo es comprender los significados esenciales del Servicio Solidario desde las experiencias vividas de aprendizaje en las Cátedras de Compromiso Social de la UCAB, un rasgo identificado en una investigación doctoral previa.

Para ilustrar la finalidad de este artículo se destacan:

- ¿Cuáles son los rasgos esenciales del aprendizaje de la es?

- ¿Cómo se manifiesta el aprendizaje de la es?

- Los estilos de vida reflejan las formas de interactuar de la sociedad con su entorno. El Laudato si" (Franciscus, 2015) menciona que "la degradación de la naturaleza está estrechamente unida a la cultura que modela la convivencia humana” (p. 3).

\section{Fundamentación teórica}

En los ods para el año 2030 destaca la meta de que todos los alumnos adquieran conocimientos teóricos y prácticos para promover el DS. La dialéctica propuesta se apoya en los cuatro pilares del aprendizaje formulados por Delors (1996) en su informe para la Unesco titulado La educación encierra un tesoro: aprender a saber, aprender a hacer, aprender a vivir juntos y aprender a ser. Posteriormente, se agregó un quinto pilar: "aprender a transformarse y a transformar la sociedad” (Unesco, 2012, p. 36) para promocionar estilos de vida sostenible, conducentes a una cultura de paz y de ciudadanía mundial.

En la última Conferencia Mundial sobre EDs, la Unesco expresó su visión humanista e integral:

Creemos que la EDS debe basarse en el respeto a la naturaleza, así como en los derechos humanos, la democracia, el estado de derecho, la no discriminación, la equidad y la igualdad de género, $y$ promoverlos. Además, debería promover el entendimiento intercultural, la diversidad cultural, una cultura de paz y no violencia, la inclusión y la noción de una ciudadanía mundial responsable y activa. (Unesco, 2021, p. 2).

En esta línea destaca el aporte de Tapia (2010) y su equipo Clayss, quienes identifican el Aprendizaje-Servicio-Solidario (APSS) cuando se "tiene simultáneamente objetivos de compromiso social y objetivos de aprendizaje evaluables, y ofrece a los estudiantes oportunidades de poner en juego no sólo valores y actitudes solidarias, sino también conocimientos y competencias específicas vinculadas a su perfil profesional" (p. 17). Esto lo reafirma Tapia (2021) en el Congreso sobre APss al lograrse todas estas competencias en un mismo proyecto, $y$ añade que esto se corresponde a las didácticas activas de la metodología por proyecto o con base en la resolución de problemas.

Por otra parte, los principios éticos del Laudato si - como "el desafío urgente de proteger nuestra Casa Común incluye la preocupación de unir a toda la familia humana en la búsqueda de un desarrollo sostenible e integral" (p. 12) — impulsan a superar actitudes de indiferencia y resignación.

Para esto, es necesaria la participación de todos y compartir como humanidad un código basado en principios bioéticos para la transformación de la sociedad: 


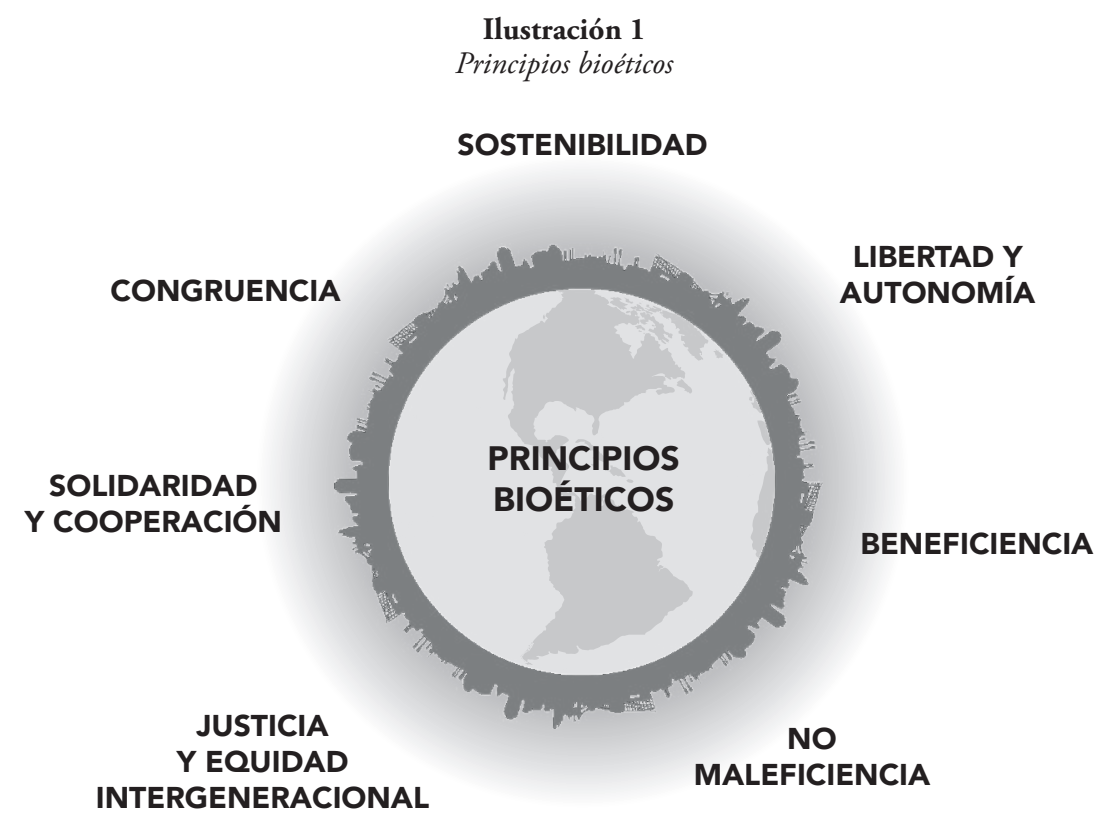

Fuente: Giraud, Méndez \& Chacón (s.f., p. 3)

Similarmente, la Carta de la Tierra (2000) representa un marco ético para la comprensión de las interrelaciones del DS, apoyado en cuatro principios interdependientes: el respeto y cuidado de la comunidad de la vida; la justicia social y económica; la integridad ecológica; y la democracia, la no violencia y la paz. Por otra parte, en el Manifiesto por la Vida (Galano et al., 2002) destacan los siguientes principios: ética de una producción para la vida; ética de la ciudadanía global; ética de los derechos, la justicia y la democracia; ética de los bienes comunes y del bien común; ética del ser y el tiempo de la sustentabilidad, entre otros.

De igual manera, la pedagogía ignaciana es orientadora de la formación universitaria, "conocer la verdad y aprender a discernir el bien llevan a ordenar la persona para la libertad y el bien común" (Ugalde, 2004, p. 135). Más recientemente, Ugalde (2018) nos interpela en cuanto a qué significa una universidad jesuita hoy: "aportamos a formar líderes multiplicadores no sólo de individuos para sí, sino hombres y mujeres para los demás" (p. 6).

Con la finalidad de darle sustento didáctico al planteamiento anterior, resulta necesario conocer los momentos sugeridos en su enfoque pedagógico:
1) Acompañar al joven a ver el contexto, conocer la realidad circundante, mirándose primero a sí mismo, sus posibilidades y destrezas para observar el entorno, el barrio, la ciudad, la región, el país, el continente, el mundo.

2) Implicarse en la experiencia educativa propicia que el acompañante y el acompañado se dejen tocar por la experiencia, que observen y reflexionen esa realidad, y en esa dinámica revisen sus sentimientos: ¿qué me reclama esa realidad?, ¿qué intereses surgen a partir de ella?, ¿qué aprendizajes obtuve en esa primera aproximación a la realidad? Duplá (2000), compilador del Cuaderno Ignaciano Núm. 1, sostiene que "el Paradigma Ignaciano, a través de la reflexión, enseña a pensar, a analizar críticamente la realidad" (p. 8), a sopesar los diversos aspectos de las cosas, a construir conocimiento.

3) La reflexión genera que los estudiantes, junto con el profesor, desarrollen técnicas de aprender a aprender y superar lo memorístico. Se acompaña al estudiante para que sienta, relacione situaciones que conoce con las que está observando, contraste conocimientos y datos anteriores con lo actualmente vivido; a partir de ahí, estará en

32 - Servir solidariamente, un rasgo esencial del aprendizaje para el desarrollo sostenible desde el compromiso social María Teresa Sánchez Reyes, Damelis del Valle Cermeño Guaina DIDAC 79 (2022): 29-38 
posición de proponer. Es interesante escuchar al profesor Najarro (2012) de la Universidad Rafael Landívar de Guatemala plantear que, según su experiencia y visión, estos momentos no son rígidos, que puede estarse dando una dinámica de reflexión en todo momento (véase https://www. youtube.com/watch?v=L5KjYG9U9i0).

4) La acción debe ser transformadora: se espera que el estudiante y su asesor dejen un impacto social en el contexto donde se está trabajando, con el propósito de mejorar el entorno que interpeló al estudiante. Incluso éste debe salir más potenciado como persona en sus valores, conocimientos y acciones. En este punto, es pertinente incluir la frase de San Ignacio, citada por Duplá: "Todo lo que hagamos debe pasar por mente, corazón y voluntad” (2000, p. 177). En este postulado se devela la relevancia de un ser humano en acción, trascender y no sólo hacer.

5) La evaluación de lo realizado. En el ambiente universitario esto genera una calificación. Ahora bien, Najarro (2012) sugiere que sea cualitativa, aplicando las habilidades y competencias cognitivas del estudiante; no repetitivas, no memorísticas ni ajustadas a lo que diga el profesor. Después de realizar la evaluación de la experiencia y del actuar, se volverá a mirar el contexto, a reflexionar sobre él, sopesar lo vivido, actuar de nuevo sobre esa realidad, evaluarla y continuar el ciclo dinámico del impactar socialmente.

Desde una perspectiva didáctica, estos momentos son dinámicos e interdependientes. Es así como la pedagogía ignaciana y la metodología de APSs son concebidas como didácticas en las cátedras de Práctica de Proyectos Comunitarios de la mención de Integral y Práctica de Ambiente No Convencional, de la mención de Preescolar de la carrera de Educación, tomadas en la muestra.

Todo esto propicia un cambio en la mirada desde una perspectiva moral e individual hacia la responsabilidad universal y se valora la ética de la solidaridad.

\section{Metodología}

Formar ciudadanos capaces de tomar decisiones con conocimiento de causas y consecuencias morales y éticas amerita desarrollar en el hombre

Un ciudadano formado se convierte en un ciudadano comprometido en resolver situaciones de su realidad y por ende con ello construirá un mundo más equilibrado y sostenible. Este ejercicio formativo implica desarrollar competencias de convivencia, desarrollo de una conducta para la paz, participación activa, responsabilidad, inclusión, respeto a las diferencias no sólo culturales sino de opinión y actuación, siempre y cuando estén en el marco de lo ético-social. (Sánchez, 2017, p. 104).

Investigar con la FH es explorar el mundo de los significados pedagógicos. Es indagar sobre la esencia, "el eidos, lo que algo es, y sin lo cual, no podría ser lo que es" (Van Manen, 2003, p. 14). En la investigación se observó el fenómeno en su ambiente natural; a través de protocolos de experiencias vividas (PEV).

El análisis de los PEv (25 anécdotas, dos entrevistas conversacionales y tres grupos focales) se abordó mediante métodos empíricos y reflexivos, propuestos por Van Manen. Los primeros traducen las experiencias vividas personalmente y las vividas por las experiencias de otros, mediante relatos anecdóticos. La anécdota como un recurso capaz de humanizar el fenómeno pedagógico, utilizando incluso lenguaje de otras áreas, como la poesía o el cine, genera reflexión de una manera amena y profunda.

Los reflexivos destacan el análisis temático, búsqueda de significados, aislamiento de afirmaciones temáticas, reducción "eidética" y hermenéutica, comprensión de significados a través de entrevista hermenéutica y aportes de otros, con el propósito de esclarecer la estructura del fenómeno (citado por Ayala, 2008). Este tipo de conocimientos es relacional, lo humano se constituye en el lenguaje, el entrelazamiento de lo emocional y lo racional, marcando un modo particular de vivir o coexistir (Maturana, 2003). 
Resultados

Aplicado con rigurosidad el método FH, seis temas reflejan los significados esenciales identificados en la investigación original.

El aprendizaje de la EDs es:

1) Visibilizar al otro.

2) Vivificar el currículo.

3) Formar en humanidades al joven educando.

4) Servir solidariamente, consciente del para qué y el para quién.

5) Valorar la dignidad humana de cara al cumplimiento de los ods.

6) Contar con docentes comprometidos, dispuestos a ser mediadores de un aprendizaje ético-social para el DS.

A los efectos del artículo se presenta el tema "Servir solidariamente, consciente del para qué y el para quién" mediante un TF con una narración "que aglutina elementos cognitivos y emotivos, descriptivos e ilustrativos, en su intención de expresar singularmente la naturaleza o esencia de cada dimensión de la experiencia vivida” (Jordán, 2015, p. 2).

$\mathrm{Al}$ respecto, Morín (1999) menciona en su publicación Los siete saberes necesarios para la educación del futuro sobre este tipo de requerimiento para la educación: "la conciencia de nuestra humanidad en esta era planetaria nos debería conducir a una solidaridad y a una conmiseración recíproca del uno para el otro, de todos para todos" (p. 42). Esto demanda aprender a ser compasivos para la comprensión de una ética para vivir.

A continuación, el TF producto de la reflexión temática en un círculo hermenéutico al estilo ignaciano.

TF: Servir solidariamente, consciente del para qué y el para quién. Rasgos esenciales

$\mathrm{Al}$ interior de la institución subyace la esperanza de inclinarse sobre el tema del Ds. En la medida que la universidad esté inmersa se vinculará a los jóvenes, empezarán a apostar al tema porque en definitiva lo importante es conseguir en esta zona respuestas del siglo XXI, es decir, la humanidad como humanidad, considerando el Ds como un tema transversal. Así se parafrasea la experiencia del docente-vicerrector, responsable de la dirección de la institución.

Esta postura requiere una relación abierta a procesos dialógicos, respetuosos, con la mirada puesta alrededor, para que los estudiantes confronten la realidad con el sentido integral de la persona, promoviendo la posibilidad de uno para el otro.

A continuación, una docente de la Escuela de Educación, a partir de una experiencia en medio de una situación sociopolítica convulsiva, asume con sus estudiantes una metodología constructivista marcada por la pedagogía ignaciana y el aprendizaje-servicio como didáctica, desde su vivencia en una cátedra de Compromiso Social:

Incluimos este tema de Derechos Humanos en las cátedras de Práctica de Proyectos Comunitarios de la mención de Integral y Práctica de Ambiente No Convencional de la mención de Preescolar [...]. Todas las estudiantes se motivaron y les pareció novedoso en la carrera, ampliar su mirada fuera del aula y conocer a profundidad los derechos de las mujeres y cómo esto evidentemente impacta en la armonía familiar [...]. Además, que una de ellas había leído que por el confinamiento producto de la pandemia parecían haber aumentado los índices de violencia intrafamiliar.

Es así como la docente promueve entre sus estudiantes dinámicas reflexivas que les permite interpretar y tomar conciencia sobre el tema de la violencia de la mujer, abriéndose a la posibilidad de una observación crítica de los comportamientos de sus niños en el aula que podría ser reflejo de indicadores de violencia en sus hogares.

En este orden de ideas, Morín (1999) plantea que un conocimiento que sea pertinente deberá considerar: el contexto, lo global, lo multidimensional y lo complejo. Este mismo autor enfatiza en la 
necesidad de promover una inteligencia general que motive la curiosidad propia de los niños, la cual suele ser mutilada con los saberes aislados especializados: "el debilitamiento de la percepción de lo global conduce al debilitamiento de la responsabilidad [...] y al debilitamiento de la solidaridad" (p. 18).

Este planteamiento se evidencia en la experiencia de la docente en el aula, ella cocrea con sus estudiantes dinámicas de aprendizaje con adolescentes y madres de comunidades cercanas, para que puedan identificar valores y cultura social proclives a un clima de violencia de la sociedad y así manejen competencias para que las futuras educadoras, desde el ejercicio profesional, contribuyan a humanizar a la sociedad.

La docente y sus estudiantes se apropian del paradigma ignaciano, la experiencia se llena de vida al dejarse "tocar" por el contexto, la reflexión se hace consciente con preguntas generadoras, desde una perspectiva constructivista del aprendizaje; se actúa y se evalúa, tal como sustenta Najarro (2012), en un proceso tan dinámico como el propio ser humano.

En consecuencia, la escucha activa, la responsabilidad ética y el tacto pedagógico aumentan la capacidad de "conversación" para encarar los problemas globales y locales, de manera que los jóvenes estén dispuestos a aprender, ordenen sus saberes y haberes para la defensa de los derechos humanos, como bien común y universal.

\section{Manifestaciones del servir solidariamente}

La EDS se manifiesta éticamente en el respeto a la libertad, de forma que el estudiante, desde el discernimiento, confronte la realidad con el sentido integral de la persona humana y el contexto circundante, como elementos integrantes para dar soluciones.

Así lo expresa la docente desde su experiencia en el aula:

Generamos distintas estrategias hasta que consideré prudente que sola no podía; se invitaron en primer momento diferentes aliados, [...] de Amnistía Internacional y [...] un conocedor del tema por ejercicio personal desde PROVEA, primero con charlas informativas y documentos que nos facilitaron los charlistas. En una segunda fase, basados en la experiencia y los comentarios reflexivos de las jóvenes, [...] salimos de la UCAB para asistir a talleres o conferencias, charlas informativas del marco legal, materiales tipo cartillas, talleres con dinámicas reflexivas. De esta interacción surgió una valiosa experiencia, las estudiantes comenzaron a tener conciencia de sus propios conceptos relacionados con el tema de la violencia a la mujer, [...] e incluso, hicieron conciencia de la posibilidad de una observación crítica de los comportamientos de sus niños en el aula, como reflejo de indicadores de violencia en sus hogares.

Según la docente, de esta manera la capacidad de "mirar" el contexto con su complejidad multidimensional permite valorar:

- La sensibilización del tema "Violencia basada en género" y el impacto en la armonía familiar y en el desarrollo del niño.

- La búsqueda de información más allá de lo trabajado en el aula.

- La Escuela de Educación sensibilizada con el tema "Violencia basada en género".

Estas experiencias de cátedras de Compromiso Social permiten que los estudiantes ofrezcan sus conocimientos adquiridos en sus carreras y al mismo tiempo aprendan en la comunidad: al dar reciben. Incluso el discernimiento de que no sólo en el aula impacta su labor docente sino, por el contrario, más allá del aula debe transformar la realidad del niño y de las familias. Esto supera el pensamiento reduccionista de un ser biológico y desarrolla personas capaces de cocrear con otros un espacio humano de convivencia social (Maturana \& Nisis, 1997).

Intimar con la realidad de su contexto procura ciudadanos que regeneren la democracia, "la regeneración del civismo supone la regeneración de la solidaridad y de la responsabilidad, es decir el desarrollo de la antropoética” (Morín, 1999, p. 63); la regeneración democrática individual o en redes, 
desde el servicio solidario, impacta en la formación de la ciudadanía. Se demostró que incluso durante una pandemia es posible desarrollar proyectos de corte social.

\section{Aproximación a un modelo ético de aprendizaje}

El aprendizaje para el Ds es mostrado desde el respeto a la interacción persona-sociedad-especie-Casa Común. Esto impactará en un modelo educativo transformador hacia un humanismo integral, dirigido a fortalecer las competencias de aprender a conocer, aprender a ser, aprender a convivir, aprender a trascender, aprender a cuidar la Casa Común.

Por lo tanto, se puede valorar la ética como el eje transversal en el proceso de enseńanza y aprendizaje, con énfasis en la pedagogía ignaciana, en un contexto interdisciplinar y transdisciplinar, como instrumentos que promoverán el aprendizaje de una Es.

Ilustración 2

Constructos de la ética para la sostenibilidad (ES)

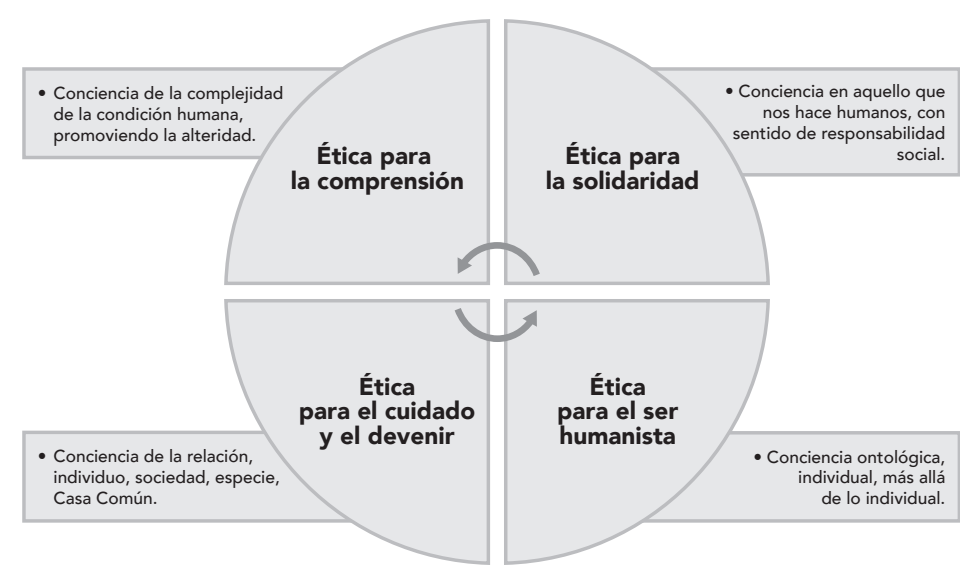

Fuente: Elaboración propia.

\section{Conclusiones}

La pedagogía ignaciana permite desarrollar una estructura de reflexión personal y colectiva, y este modo de actuar garantiza la formación de un profesional integral, comprometido éticamente desde el discernimiento, su hacer, el saber y el convivir.

La EDS apoyada en la pedagogía ignaciana promueve experiencias cercanas y amorosas para que los jóvenes se convenzan de que, como resultado de una elección ética, hay otros modos de hacer las cosas: modos más cercanos al otro, acompañarlo en las experiencias; observar el contexto y aportar para su transformación; al mismo tiempo, los jóvenes se apropian de un estilo de actuar como profesionales y como personas.

Empoderar a los jóvenes como agentes multiplicadores del Ds es clave. Sosa (2019) expresa que "son los jóvenes, con su perspectiva, quienes pueden ayudarnos a comprender mejor el cambio de época que estamos viviendo y su novedad esperanzadora" (p. 4). Esto se enriquece con estrategias experienciales.

\section{REFERENCIAS}

Ayala, R. (2008). La metodología fenomenológico-hermenéutica de M. Van Manen en el campo de la investigación educativa. Posibilidades y primeras experiencias. Revista de Investigación Educativa, 26(2), 409-430. Recuperado de https:/www.redalyc.org/pdf/2833/283321909008.pdf

Ayala, R. (2011). La esperanza pedagógica. Una mirada fresca y profunda a la experiencia educativa desde el enfoque de Van Manen. Revista española de pedagogía, LXIX(248),
119-144. Recuperado de https://revistadepedagogia.org/ wp-content/uploads/2011/01/248-007.pdf.

Comisión de la Carta de la Tierra. (2000). Carta de la Tierra. Holanda: Unesco. Recuperado de http://cartadelatierra.org/

Delors, J. (1996). La educación encierra un tesoro. Informe a la Unesco de la Comisión Internacional sobre la educación para el siglo XXI. París: Santillana.

Fernández, D. (2005). La Universidad de la Compañía de Jesús. Su identidad y espiritualidad (elementos para su cons-

36 - Servir solidariamente, un rasgo esencial del aprendizaje para el desarrollo sostenible desde el compromiso social María Teresa Sánchez Reyes, Damelis del Valle Cermeño Guaina DIDAC 79 (2022): 29-38 
trucción). En S. Di Trolio (Comp.), Identidad ignaciana y universidad (pp. 155-170). Caracas: Publicaciones UCAB.

Franciscus (2015). Laudato si'. Vaticano II, Roma. Recuperado dehttp://w2.vatican.va/content/francesco/es/encyclicals/ documents/papa-francesco_20150524_enciclica-laudatosi.pdf

Galano, C., Curi, M., Motomura, O., Porto, C., Silva. M. et al. (2002). Manifiesto por la vida. Por una ética para la sustentabilidad. Ambiente \& Sociedade, V(10). Recuperado de www.scielo.br/scielo.php?script=sci_arttext\&pi$\mathrm{d}=$ S1414-753X2002000100012

Giraud, L., Méndez, D. \& Chacón, R. (s. f.). Hacia una ética del desarrollo sostenible. Caracas: Academia Nacional de Ingeniería y Hábitat. Comisión de Ambiente. Recuperado de www.acading.org.ve/...Etica/Hacia_una_Etica_del_Desarrollo_Sostenible.

Jordán S., J. A. (2015). La responsabilidad ética-pedagógica de los profesores-educadores: una mirada nueva desde una perspectiva ética Max van Manen. Revista Española de Pedagogía, LXXIII(261), 381-396. Barcelona: Universidad de Barcelona. Recuperado de https://revistadepedagogia. org/wp-content/uploads/2015/06/la-responsabilidadetica-pedagogica.pdf

Kolvenbach. (2000). Discurso P. General. Santa Clara. Recuperado de Javeriana.edu.co

Manen, M. van (2003). Investigación educativa y experiencia vivida. Ciencia humana para una pedagogía de la acción y la sensibilidad. Barcelona: Idea Books. Recuperado de https://dialnet.unirioja.es/servlet/libro? codigo=52958

Maturana, H. (2003). Ontología del conversar. En J. Luzoro (Comp.), Desde la biología a la psicología (pp. 86-110). Buenos Aires: Lumen.

Maturana, H. \& Nisis, S. (1997). Formación humana y Capacitación. Fondo de las Naciones Unidas para la Infancia (Unicef). Santiago de Chile: Dolmen Ediciones.

Morín, É. (1999). Los siete saberes necesarios para la educación del futuro. París: Unesco. Recuperado de http://www.ideassonline.org/public/pdf/LosSieteSaberesNecesariosParaLaEdudelFuturo.pdf

Najarro, A. (2012). Mensaje del artículo "Universidad y pedagogía ignaciana”, del M. A. Armando Najarro. Boletin de Formación Docente. Recuperado de https://www.youtube.com/watch?v=L5KjYG9U9i0
Organización de las Naciones Unidas para la Educación, la Ciencia y la Cultura, (Unesco). (2012). Educación para el Desarrollo Sostenible (libro de consulta). Serie Instrumentos de aprendizaje y formación Núm. 4. Recuperado de UNESCO.org/images/0021/002167/216756s.pdf

Organización de las Naciones Unidas para la Educación, la Ciencia y la Cultura (Unesco). (2021). Declaración de Berlin sobre la Educación para el Desarrollo Sostenible. Recuperado de https://en.unesco.org/sites/default/files/esdfor2030berlin-declaration-es.pdf

Sánchez, M. (2017). Trabajo en Red, Andragogia, Capital Social y Sustentabilidad. Redes Comunitarias para el Tejido Social. Madrid: Editorial Académica Española.

Sosa, A. (2019). Preferencias Apostólicas Universales de la Compañia de Jesús. Recuperado de www.jesuits.global

Tapia, M. N. (2010). La propuesta Pedagógica del Aprendizaje Servicio. Una propuesta latinoamericana. Tzhoecoen. Revista Cientifica, 5, 23-43.

Tapia, M. N. (2021). Conferencia Inaugural. 24. ${ }^{\circ}$ Seminario Internacional de Aprendizaje Servicio Solidario. Canal YouTube Clayss. Recuperado de https://www.youtube.com/watch?v=ArDgGkNZ1a0

Ugalde, L. (2004). Letras y espíritu. Desafíos de la educación universitaria. En S. Di Trolio (Comp.), Identidad ignaciana y universidad (pp. 125-140). Caracas: Publicaciones UCAB.

Ugalde, L. (2018). ¿Qué significa la Universidad Jesuita hoy en América Latina? [Discurso principal]. Conferencia en la Universidad Iberoamericana León, México. Recuperado de https://issuu.com/ausjal/docs/discurso_ibero_leon

Universidad Católica Andrés Bello (UCAB). (2013). Proyecto Formativo Institucional -PFI. Caracas: Publicaciones UCAB. Recuperado de http://w2.ucab.edu.ve/proyecto-formativoinstitucional.html

Universidad Católica Andrés Bello (UCAB). (2017). Encuesta Cultura de Sustentabilidad 2014-2016 (no publicado). Caracas: Publicaciones UCAB.

Universidad Católica Andrés Bello (UCAB). (2019). Resultados Cultura de Sustentabilidad Caracas-Guayana. Año lectivo 207-2018 (no publicado). Caracas: Publicaciones UCAB.

Universidad Católica Andrés Bello (UCAB). (2020). Reglamento de Extensión Social Universitaria. Núm. 2.59. Caracas: Publicaciones UCAB. Recuperado de https://www.ucab.edu. ve/wp-content/uploads/sites/2/2020/03/2.59-modificado-3-03-2020.pdf

BIBLIOGRAFÍA RECOMENDADA

Agúndez, M. (2008). El paradigma universitario Ledesma-Kolvenbach. Revista de Fomento Social, 63, 603-631. Recuperado de https://www.javeriana.edu.co/cardoner/wp-content /uploads/2019/08/paradigma-ledesma-kolvenbach-agundez-2008.pdf

Asociación de Colegios Jesuitas de Colombia (2009). El P. Peter-Hans Kolvenbach y la educación. 1983-2007 (Selección de escritos). Bogotá: Editorial Kimpress Ltda.

CLAYss Digital. (2021). Apertura del 24. o Seminario Internacional Aprendizaje Servicio Solidario. [Conferencia Inau- gural: María Nieves Tapia]. Recuperado de https://www.youtube.com/watch?v=ArDgGkNZ1a0

Compañía de Jesús (2000). Pedagogía ignaciana. Tres documentos contemporáneos. Caracas: Publicaciones UCAB.

Fuster, G. \& Doris, E. (2019). Investigación cualitativa: Método fenomenológico hermenéutico. Propósitos y Representaciones, $7(1), 202-229$.

Ruíz A., S., Valenzuela, T. \& Rodríguez, R. (2014). La pedagogía ignaciana y la evaluación. Managua: Universidad Centroamericana UCA. 


\section{SEMBLANZAS}

Maria Teresa Sánchez Reyes. Doctora en Ciencias de la Educación. Mención Andragogía por la Universidad Interamericana de Educación a Distancia de Panamá. Profesora

Universitaria (desde 1999 a la actualidad) en la UCAB. Desde 2009 es directora de Extensión Social Universitaria. Entre sus líneas de investigación se encuentran: redes sociales comunitarias; pedagogía social; aprendizaje servicio. Hizo una estancia académica en la Universidad Iberoamericana Ciudad de México del 1 de febrero de 2019 al 30 de marzo de 2019, así como en el Instituto de Investigaciones para el Desarrollo de la Educación (INIDE). Algunas de sus publicaciones recientes son el artículo "Propuestas para la educación rural en Venezuela”, en el portal Educación Futura: http://www. educacionfutura.org/la-educacion-rural-en-venezuela-propuestas-de-mejora/; y el libro

Trabajo en Red, Andragogía, Capital Social y Sustentabilidad. Redes Comunitarias para el Tejido Social.

Damelis del Valle Cermeño Guaina. Doctora en Educación. Mención Educación para el Desarrollo Sustentable por la UCAB. Especialista en Ciencias AdministrativasMención Finanzas. Su estudio de pregrado es en Ingeniería Industrial. Es profesora universitaria desde 2001 en la UCAB y coordinadora de Auditoría Interna en esta misma institución desde 2018. Obtuvo el reconocimiento como Primer Lugar de Investigación en la UCAB, 2019-2020. Su línea de investigación se basa en la educación para el desarrollo sostenible-ética de la sostenibilidad. Entre sus últimas publicaciones se encuentran un artículo en la Revista Digital Guayana Moderna y su tesis doctoral El aprendizaje para el desarrollo sustentable como experiencia vivida en la UCAB. Un acercamiento a un nuevo modelo ético-social, la cual obtuvo la Mención Publicación. UCAB. Doctorado en Educación. Fue invitada como conferencista al Pre-II Simposio Venezolano de Ciudades Inteligentes y Sostenibles Ciudad Guayana 2021. 